\title{
miR-23b-3p induces the cellular metabolic memory of high glucose in diabetic retinopathy through a SIRT1-dependent signalling pathway
}

\author{
Shuzhi Zhao ${ }^{1}$ - Tao $\mathrm{Li}^{1}$ • Jun $\mathrm{Li}^{2}$ • Qianyi Lu ${ }^{1}$ Changjing Han ${ }^{1}$ - Na Wang ${ }^{1}$. \\ Qinghua Qiu ${ }^{1} \cdot$ Hui $\mathrm{Cao}^{1} \cdot \mathrm{Xun}_{\mathrm{Xu}^{1}}{ }^{1}$ Haibing $\mathrm{Chen}^{3} \cdot \mathrm{Zhi}^{\mathrm{Z}} \mathrm{Hheng}^{1}$
}

Received: 18 July 2015 / Accepted: 17 November 2015 /Published online: 19 December 2015

(C) Springer-Verlag Berlin Heidelberg 2015

\begin{abstract}
Aims/hypothesis The mechanisms underlying the cellular metabolic memory induced by high glucose remain unclear. Here, we sought to determine the effects of microRNAs (miRNAs) on metabolic memory in diabetic retinopathy. Methods The miRNA microarray was used to examine human retinal endothelial cells (HRECs) following exposure to normal glucose $(\mathrm{N})$ or high glucose $(\mathrm{H})$ for 1 week or transient $\mathrm{H}$ for 2 days followed by $\mathrm{N}$ for another 5 days $(\mathrm{H} \rightarrow \mathrm{N})$. Levels of sirtuin 1 (SIRT1) and acetylated-nuclear factor $\mathrm{kB}$ (Ac-NF- $k B$ ) were examined following transfection with miR-23b-3p inhibitor or with SIRT1 small interfering (si)RNA in the $\mathrm{H} \rightarrow \mathrm{N}$ group, and the apoptotic HRECs were determined by flow cytometry. Retinal tissues from diabetic rats were similarly studied following intravitreal injection of miR-23b-3p inhibitor. Chromatin immunoprecipitation (ChIP) analysis was performed to detect binding of NF-KB
\end{abstract}

Electronic supplementary material The online version of this article (doi:10.1007/s00125-015-3832-0) contains peer-reviewed but unedited supplementary material, which is available to authorised users.

Haibing Chen

chenhb@sjtu.edu.cn

Zhi Zheng

zzheng88@sjtu.edu.cn

1 Department of Ophthalmology, Shanghai First People's Hospital Affiliated to Shanghai Jiao Tong University, Haining Road 100, Shanghai 200080, People's Republic of China

2 Department of Ophthalmology, Lishui City Center Hospital, Lishui, People's Republic of China

3 Department of Endocrinology and Metabolism, Shanghai Sixth People's Hospital Affiliated to Shanghai Jiao Tong University, Yishan Road 301, Shanghai 200233, People's Republic of China p65 to the potential binding site of the $m i R-23 b-27 b-24-1$ gene promoter in HRECs.

Results High glucose increased miR-23b-3p expression, even after the return to normal glucose. Luciferase assays identified SIRT1 as a target mRNA of miR-23b-3p. Reduced miR-23b-3p expression inhibited Ac-NF- $\mathrm{kB}$ expression by rescuing SIRT1 expression and also relieved the effect of metabolic memory induced by high glucose in HRECs. The results were confirmed in the retina using a diabetic rat model of metabolic memory. High glucose facilitated the recruitment of NF-KB p65 and promoted transcription of the miR-23b-27b-24-1 gene, which can be suppressed by decreasing miR-23b-3p expression.

Conclusions/interpretation These studies identify a novel mechanism whereby miR-23b-3p regulates high-glucoseinduced cellular metabolic memory in diabetic retinopathy through a SIRT1-dependent signalling pathway.

Keywords Diabetic retinopathy - Metabolic memory · miR-23b-3p $\cdot$ NF-kB $\cdot$ SIRT1

\begin{tabular}{ll}
\multicolumn{2}{l}{ Abbreviations } \\
Ac-NF-kB & Acetylated-NF-kB \\
ChIP & Chromatin immunoprecipitation \\
H & High glucose group \\
HREC & Human retinal endothelial cell \\
miRNA & MicroRNA \\
MN & Mannitol + normal glucose group \\
N & Normal glucose group \\
NF-kB & Nuclear factor kB \\
q(RT-PCR) & Quantitative (RT-PCR) \\
si(RNA) & Small interfering (RNA) \\
SIRT1 & Sirtuin 1 \\
STZ & Streptozotocin
\end{tabular}




\section{3'-UTR 3'-untranslated region \\ WT Wild type \\ NC Normal control \\ LNA Locked nucleic acid \\ ISH In situ hybridisation}

\section{Introduction}

Diabetic retinopathy is a debilitating vascular complication of diabetes that occurs primarily through the long-term detrimental effects of hyperglycaemia [1]. Vascular complications often persist and may progress despite improved glucose control, suggesting that previous episodes of transient hyperglycaemia may induce longstanding deleterious vascular dysfunction [2]. This phenomenon has been defined as 'metabolic memory' $[2,3]$ and remains poorly understood and a major challenge in treating diabetes.

Accumulating studies have reported the altered expression of inflammatory genes during diabetic retinopathy [4]. These retinal inflammatory mediators may be activated for several months after the reinstitution of glycaemic control following a period of poor control $[5,6]$. Nuclear factor $\kappa B(N F-\kappa B)$, which is activated by high glucose and promotes the expression of pro-inflammatory cytokines and pro-apoptosis regulators, has been proposed to play a role in the vascular complications of diabetes [7].

Sirtuin 1 (SIRT1), an $\mathrm{NAD}^{+}$-dependent class III histone deacetylase, plays critical roles in stress responses, metabolism, cell cycle and survival [8-10]. It regulates cellular inflammation in endothelial cells, and the anti-inflammatory property of SIRT1 is closely related to its inhibition of NF-kB [11-13].

Sirtuin 1 confers resistance to the high-glucoseinduced cellular metabolic memory through an inflammatory signalling pathway in diabetic retinopathy [14]. However, the mechanisms of SIRT1 regulation remain incompletely understood.

MicroRNAs (miRNAs) represent an exciting area of research because of their involvement in many biological processes, including the development of metabolic diseases such as diabetes [15-17]. As highly conserved, short non-coding RNAs (21-25 nucleotides in length), miRNAs negatively regulate gene expression at the post-transcriptional level by binding to the $3^{\prime}$ untranslated region (3'-UTR) of target mRNA, leading to its degradation or translational repression [17]. Several miRNAs regulate SIRT1 in various disease conditions, including diabetic retinopathy [18-20]. However, no studies have focused on the regulation of SIRT1 in the cellular metabolic memory of diabetic retinopathy.

\section{Methods}

Cell culture and treatments Cultured HRECs of three to four passages were plated at $2,500 \mathrm{cells} / \mathrm{cm}^{2}$ in six-well plates (Corning, Acton, MA, USA) and treated with normal glucose $(5 \mathrm{mmol} / \mathrm{l})(\mathrm{N})$ or high glucose $(25 \mathrm{mmol} / \mathrm{l})(\mathrm{H})$ for 1 week or $\mathrm{H}$ for $48 \mathrm{~h}$ followed by $\mathrm{N}$ for 5 days $(\mathrm{H} \rightarrow \mathrm{N})$. Cells were incubated in $20 \mathrm{mmol} / \mathrm{l}$ mannitol together with $\mathrm{N}(\mathrm{MN})$ as osmotic controls. See the electronic supplementary material (ESM) Methods for details.

Microarray and quantitative RT-PCR analysis of miRNA The HREC miRNA expression profiles were compared using miRNA microarray analysis following exposure to normal glucose, high glucose, or transient high glucose followed by normal glucose. The results were verified by quantitative (q)RT-PCR analysis. See the ESM Methods for details.

Molecular biology methods Total RNA was extracted from cultured cells and tissues using TRIzol (Invitrogen, Carlsbad, CA, USA) for mRNA analyses. Levels of SIRTI mRNA were quantified by qRT-PCR using the Quantitect SYBR Green PCR Kit (Qiagen, Hilden, Germany) and normalised to $\beta$-actin. See the ESM Methods for details. Antibodies against SIRT1, NF-kB p65 (acetyl K310) or albumin were used for western blot analysis, with $\beta$-actin as an internal control. See the ESM Methods for details.

A luciferase reporter assay was performed to detect the direct regulation of the SIRT1 3'-UTR by miR-23b-3p. See the ESM Methods for further details. The deacetylase activity of SIRT1 was assessed in the nuclear fraction using a fluorometric method in accordance with the manufacturer's instructions. See the ESM Methods for details. Synthetic miR-23b-3p mimic, inhibitor or small interfering (si)RNA against SIRT1 was used for each transfection. See the ESM Methods for details. Cell apoptosis was detected with an Annexin V-FITC kit and by flow cytometry analysis (BD FACSVantage; BD Sciences, San Jose, CA, USA). The data were analysed using Cell-Quest software (BD Sciences) to determine the rate of apoptosis in the upper and lower right quadrants. See the ESM Methods for details.

Chromatin immunoprecipitation (ChIP) analysis was performed on the protein-DNA complexes isolated from crosslinked cells by immunoprecipitation with an anti-NF-kB p65 antibody (acetyl K310), and the SIRT1-binding region of the $m i R-23 b-27 b-24-1$ promoter was quantified by SYBR Greenbased qPCR. See the ESM Methods for further details.

Animal experiments Male Sprague-Dawley (SD) rats ( $\sim 200 \mathrm{~g}, 8$ weeks old) were housed in standard plastic rodent cages and maintained at a regulated environment $\left(24^{\circ} \mathrm{C}, 12 \mathrm{~h}\right.$ light, $12 \mathrm{~h}$ dark cycle with lights on at 07:00 and off at 19:00 hours). The rats were randomised into five groups $(n=10$ 
rats/group). In situ hybridisation was used to detect miR-23b$3 p$ expression in retinal tissue sections. Retinal digest procedures were performed to measure the acellular capillaries in trypsin-digested retinal blood vessels as described previously [14]. Details are in the ESM Methods. All animal protocols were approved by the Animal Care and Use Committee of Shanghai Jiaotong University Affiliated First People's Hospital.

Statistical analysis Data are presented as the mean \pm SD from at least three independent experiments. Statistical comparisons between two groups were made using a two-tailed Student's $t$ test. Differences among three or more than three groups were compared by one-way ANOVA followed by least significant difference (LSD) post hoc test. (GraphPad Prism 4.0, GraphPad, San Diego, CA, USA and SPSS version 17.0, SPSS, Chicago, IL, USA). All $p$ values were two-sided, and $p<0.05$ was considered statistically significant.

\section{Results}

\section{Screening of SIRT1-targeting miRNAs in diabetic metabolic memory in HRECs}

Using an miRNA microarray analysis, we found a total of 114 known human miRNAs with signals of more than 500 in both the $\mathrm{H}$ and $\mathrm{H} \rightarrow \mathrm{N}$ groups (ESM Table 1). Twenty-two miRNAs were upregulated by 1.5 -fold or more in both the $\mathrm{H}$ and $\mathrm{H} \rightarrow \mathrm{N}$ groups compared with the $\mathrm{N}$ group (Fig. 1a and ESM Table 2). We then used the StarBase website [21], a comprehensive web-based bioinformatics algorithm, to identify 36 potential SIRT1-binding miRNAs based on complementarities of the 3'UTR SIRT1 mRNA nucleotide sequence from at least two of five prediction tools included on the website (ESM Table 3). The level of miR-23b-3p was upregulated in HRECs after high-glucose treatment or transient high glucose followed by normal glucose and miR-23b-3p was an in silico-predicted SIRT1-targeting miRNA (Fig. 1b). In addition, we performed qRT-PCR analysis of miR-23b-3p expression in the three HREC groups as well as HRECs treated with $5 \mathrm{mmol} / \mathrm{l}$ glucose plus $20 \mathrm{mmol} / \mathrm{l}$ mannitol. The results showed the same trend as the results of miRNA microarray analysis in miR23b-3p expression, with no effects of osmolarity (Fig. 1c).

SIRT1 is a direct target of miR-23b-3p in HRECs To assess whether miR-23b-3p can directly target SIRT1, a luciferase reporter plasmid was constructed that contained either a wild-type (WT) or mutant version of the predicted interaction region in the SIRT1 3'-UTR region (Fig. 2a). Transient cotransfection of HRECs with the WT reporter plasmid and miR-23b-3p mimic led to a decrease in luciferase activity compared with the mimic control sequence. In contrast,
miR-23b-3p did not suppress the luciferase activity of the mutant reporter (Fig. 2b). This reduction of SIRT1 at the mRNA and protein levels was detected in HRECs that were transfected with ectopic miR-23b-3p (Fig. 2c-e). These results confirmed the direct regulation of SIRT1 by miR-23b-3p in HRECs.

High-glucose-induced SIRT1 reduction and cellular metabolic memory in HRECs is mediated by miR-23b-3p Cells were exposed to normal glucose, high glucose or transient high glucose followed by normal glucose with the transfection of miR-23b-3p inhibitor or control scrambled miRNA.

High-glucose treatment for 1 week resulted in significantly decreased levels of SIRT1 mRNA (Fig. 3a), SIRT1 protein (Fig. 3b, c) and enzyme activity (Fig. 3d). Compared with exposure to continuous normal glucose, SIRT1 levels were also lower in cells treated with high glucose for 2 days followed by normal glucose for 5 days (Fig. $3 b, c ; H \rightarrow N$ ). The transfection of miR-23b-3p inhibitor effectively rescued highglucose-induced SIRT1 downregulation, whereas no such effect was observed with osmolarity treatment or the transfection of scrambled miRNA.

We also measured the level of cellular apoptosis using flow cytometry analysis (Fig. 3e). Continuous exposure to high glucose for 1 week significantly increased cellular apoptosis. Apoptosis was also increased in $\mathrm{H} \rightarrow \mathrm{N}$ cells. Furthermore, transfection of miR-23b-3p inhibitor prevented the cellular apoptosis associated with high-glucose-induced metabolic memory in HRECs, and this effect was reversed by the transfection of SIRT1 siRNA (Fig. 3e, f). To further verify that the transient exposure to high glucose (2 days) induced cellular metabolic memory in HRECs, we prolonged the high-glucose exposure time to 1 week and the total treatment time to 3 weeks, as in our previous study [14]. The results were consistent with the original test using the shorter high-glucose exposure time (ESM Fig. 1). These results support the hypothesis that miR-23b-3p mediates the high-glucose-induced SIRT1 reduction and cellular metabolic memory in HRECs.

High-glucose-induced Ac-NF- $\mathrm{KB}$ expression is mediated by miR-23b-3p through SIRT1 in HRECs We performed western blot analysis to examine the Ac-NF-KB expression levels in cells exposed to normal-, high- or transiently highglucose conditions, together with transfection of miR-23b-3p inhibitor or SIRT1 siRNA. As shown in Fig. 4a, b, continuous high-glucose treatment for 1 week significantly increased AcNF- $\mathrm{KB}$ levels. Levels of Ac-NF- $\mathrm{KB}$ were also increased in cells treated with transiently high glucose compared with exposure to normal glucose. However, miR-23b-3p inhibitor transfection significantly suppressed the Ac-NF- $\mathrm{kB}$ expression induced by high glucose after glucose normalisation in HRECs. No such effect was observed with osmolarity treatment or the transfection of scrambled miRNA. 


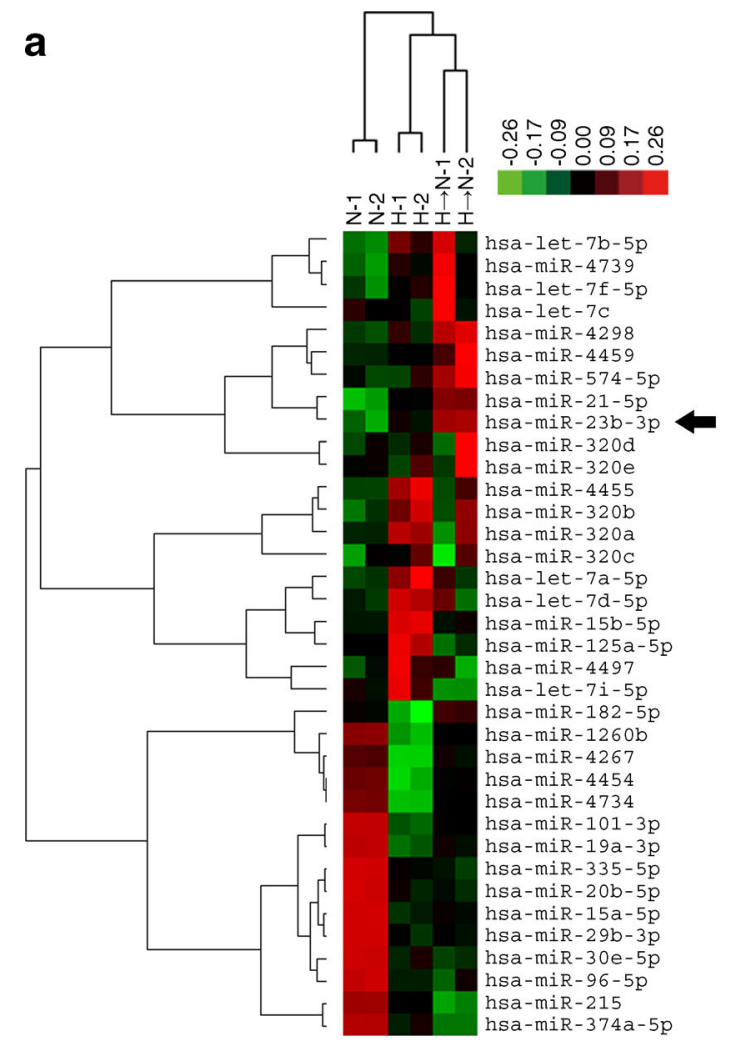

b
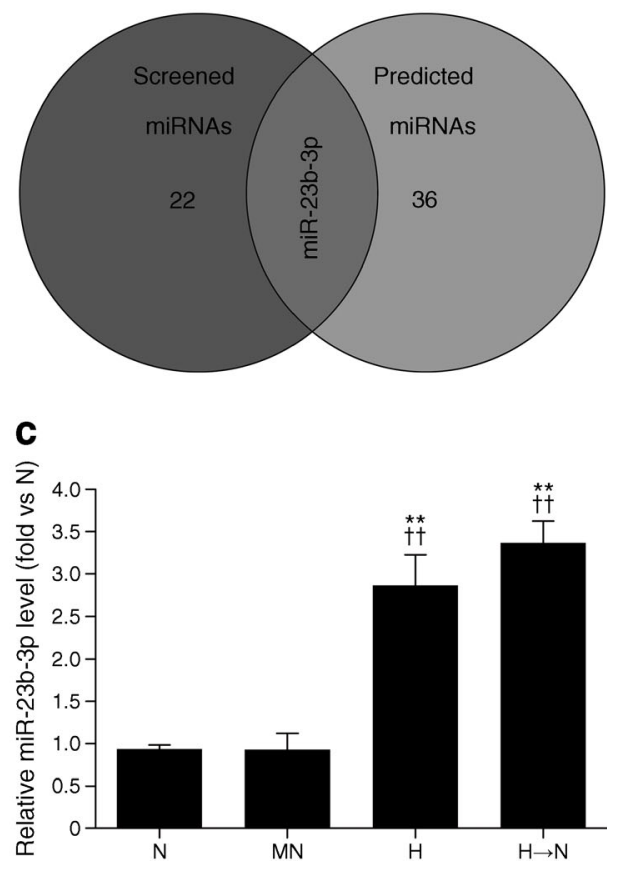

Fig. 1 High glucose increases the expression of miR-23b-3p in HRECs. (a) Thirty-six miRNAs were used for clustering those with expression levels $>1.5$ or $<0.5$ in the $\mathrm{H}$ and $\mathrm{H} \rightarrow \mathrm{N}$ groups compared with the $\mathrm{N}$ group. Hsa-, human. (b) The Venn diagram represents the simultaneously upregulated miRNAs in $\mathrm{H}$ and $\mathrm{H} \rightarrow \mathrm{N}$ using the miRNA expression microarray analysis and predicted SIRT1-targeting miRNAs by StarBase. (c)

Further transfection of HRECs with SIRT1-specific siRNA was carried out to decrease SIRT1 expression following miR$23 b-3 p$ inhibitor transfection. The repressive effect of miR23b-3p inhibitor on high-glucose-induced Ac-NF- $\mathrm{kB}$ protein expression was abolished by SIRT1 knockdown (Fig. 4a, b). Therefore, these results indicate that miR-23b-3p mediates high-glucose-induced Ac-NF- $\mathrm{kB}$ expression and metabolic memory in HRECs through the suppression of SIRT1.

\section{Memory of hyperglycaemic stress in the retinas of diabetic} animals is promoted by miR-23b-3p To determine whether these in vitro findings are associated with the development of retinal microangiopathy, an in vivo STZ-induced diabetic rat model was established. Analysis of retinal tissues from these rats with qRT-PCR after 3 months of uncontrolled diabetes showed a significant upregulation of miR-23b-3p that lasted for 3 months, even after return to normal glycaemic control (Fig. 5a). This result was further confirmed by in situ hybridisation using locked nucleic acid (LNA) probes. In the retinas of diabetic animals, miR-23b-3p was overexpressed in the cells of the vascular endothelium and the outer nuclear layers (Fig. 5b). We investigated the retinal SIRT1 and Ac-
Analysis of miR-23b-3p expression using qRT-PCR in N, MN, H and $\mathrm{H} \rightarrow \mathrm{N}$ HRECs. The results are the mean $\pm \mathrm{SD}$ of $2^{-\Delta \Delta \mathrm{C}_{\mathrm{t}}}$, the value normalised to RNU6 and expressed as fold relative to the level in one $\mathrm{N}$ HREC group from three separate experiments; ${ }^{* *} p<0.01$ vs $\mathrm{N},{ }^{\dagger \dagger} p<0.01$ vs MN

NF- $\mathrm{KB}$ protein levels. As a target of miR-23b-3p, SIRT1 protein was reduced in the retinas of diabetic rats. Conversely, Ac-NF-kB expression was increased in retinas with hyperglycaemic stress (Fig. 5c, d).

To evaluate a potential cause-effect relationship, we administered an intravitreal injection of miR-23b-3p inhibitor. The intraretinal delivery efficiency was assessed by measuring miR-23b-3p expression in the retina, and the results showed a reduction in retinal miR-23b-3p expression compared with the inhibitor normal control (NC)-injected group (Fig. 5a). This intravitreal injection of miR-23b-3p inhibitor normalised the diabetes-induced changes in SIRT1 and Ac-NF-KB expression in the retinas of treated rats. No such effect was observed following the injection of the inhibitor NC miRNA (Fig. 5c, d). Furthermore, an increase in retinal vascular permeability was detected in diabetic rats. All of the changes induced by hyperglycaemia remained for 3 months after glucose normalisation compared with exposure to continuous normal glucose. However, intravitreal injection of miR-23b-3p inhibitor significantly suppressed the vascular permeability in the retinas of rats with glucose normalisation for 3 months following 3 months of hyperglycaemia (Fig. 5e-h). 
a

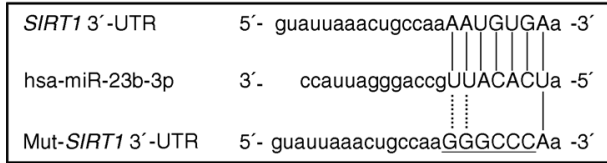

C

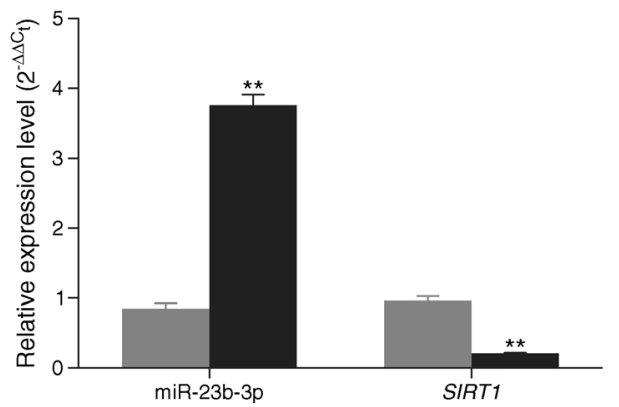

Fig. 2 SIRT1 is a validated target of miR-23b-3p. (a) Base-pair comparison between mature miR-23b-3p and the WT or mutant putative target site in the 3'-UTR of SIRT1 mRNA. The mutated binding site used for the luciferase assay is underlined. Hsa-, human. (b) Luciferase activity with various reporters in the presence or absence of miR-23b-3p mimic in HRECs. $* * p<0.01$ vs the other three groups. (c) Quantitative RT-PCR analysis of SIRT1 mRNA in cells transfected with miR-

Binding of the NF- $\mathrm{KB}$ p65 subunit to promoter elements is involved in the high-glucose-induced transactivation of miR-23b-3p in HRECs Most miRNAs are initially transcribed as primary transcripts by RNA polymerase II (Pol II) in the nucleus and can be regulated by transcriptional factors [22] such as NF- $\mathrm{kB}$, signal transducer and activator of transcription 3 (acute-phase response factor) (STAT3) and v-myc avian myelocytomatosis viral oncogene homolog (c-Myc) [23-25]. High-glucose-induced miR-23b-3p increased the expression of Ac-NF-kB by inhibiting SIRT1 in HRECs. Therefore, we hypothesised that NF-KB activation would in turn upregulate miR-23b-3p through self-stabilising circuits. A putative NF-KB p65 binding site in the $m i R-23 b-3 p$ promoter corresponding to the coding gene $m i R-23 b-27 b-24-1$ has previously been verified [23] (Fig. 6a).

To test the role of NF- $\mathrm{KB}$ p65 in high-glucose-induced miR-23b-3p expression, the specific inhibitor of kappa light polypeptide gene enhancer in $\mathrm{B}$ cells, kinase $\beta$ (IKK2) inhibitor SC-514 was added to inhibit the p65-associated transcriptional activation of the NF-kB pathway. Subsequent qRT-PCR analysis revealed the significant inhibitory effect of SC-514 on the expression of pri-miR-23b-27b-24-1 in HRECs b

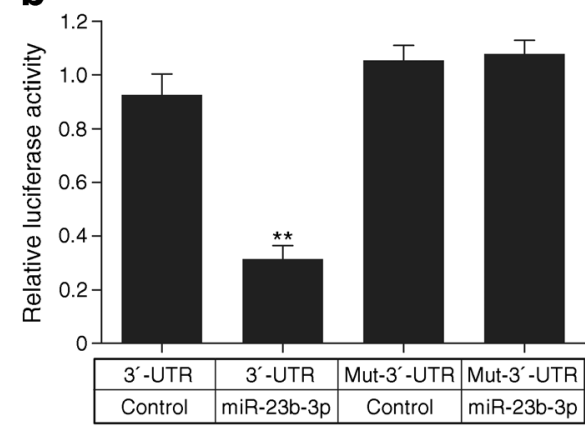

d
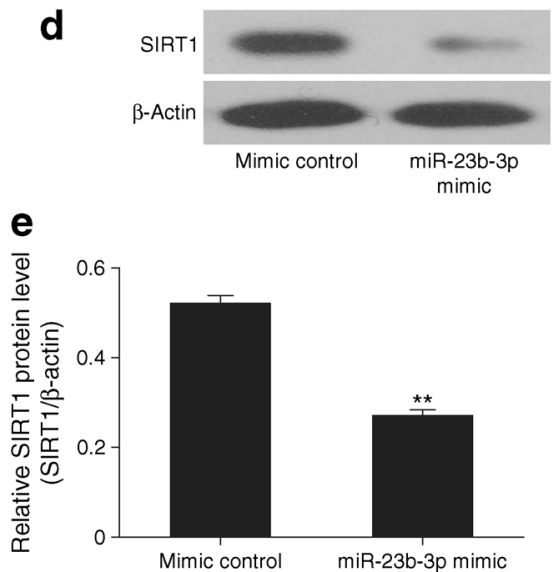

23b-3p mimic or a negative control. Grey bars, mimic control; black bars, miR-23b-3p mimic. Results are the mean \pm SD and fold relative to the level in one control group from three separate experiments. (d, e) Western blots of SIRT1 in HRECs transfected with miR-23b-3p mimic. $\beta$-actin was used as a loading control. Bar, mean $\pm \mathrm{SD} ;{ }^{* *} p<0.01$ vs the respective negative control groups. Mut, mutant

exposed to high glucose followed by normal glucose compared with cells that were not treated with SC-514 (Fig. 6b). We further performed ChIP analysis to identify the binding of the $\mathrm{p} 65$ components of NF- $\mathrm{KB}$ to the putative binding sites in the promoter elements of pri-miR-23b-27b-24-1 (Fig. 6c). Our data suggest that $\mathrm{p} 65$ binding to the promoter element mediates pri-miR-23b-27b-24-1 upregulation in high-glucose-induced transactivation of miR-23b-3p in HRECs.

\section{Discussion}

A growing number of studies have demonstrated that the molecular and pathological features of diabetic microvascular complications, including diabetic retinopathy, do not stop in response to excellent blood glucose control [26, 27]. Instead, progression continues beyond the point when good control is achieved, suggesting a 'metabolic memory' phenomenon [28, 29].

Inflammation plays an important role throughout the pathogenesis of diabetic microvascular complications [28, 30, 31]. Kowluru et al showed that high glucose significantly increased the activated caspase- 3 and NF- $\mathrm{kB}$ levels, which remained 
Fig. 3 Expression of miR-23b$3 p$ and SIRT1 and their roles in apoptosis in HRECs after culture in $\mathrm{H}$ and then $\mathrm{N}$ conditions. Quantitative RT-PCR analysis of miR-23b-3p (a), western blot (b) and quantification (c) of SIRT1 expression and activity (d) in the following cell treatment groups: $\mathrm{N}, \mathrm{MN}, \mathrm{H}, \mathrm{H} \rightarrow \mathrm{N}, \mathrm{H} \rightarrow \mathrm{N}$ plus miR-23b-3p inhibitor $(\mathrm{H} \rightarrow \mathrm{N}+$ $23 \mathrm{~b}$ In) and inhibitor $\mathrm{NC}(\mathrm{H} \rightarrow \mathrm{N}+$ In NC). (e, f) Representative cytograms and quantification showing different apoptosis levels of HRECs in the six groups plus $\mathrm{H} \rightarrow \mathrm{N}+$ miR-23b-3p inhibitor and SIRTI siRNA $(\mathrm{H} \rightarrow \mathrm{N}+23 \mathrm{~b}$ In+si-SIRT1). Results are mean \pm $\mathrm{SD}$ and fold relative to the level in one $\mathrm{N}$ HREC group $(/ \mathrm{N})$ from three separate experiments; $* * p<0.01 \mathrm{vs} \mathrm{N},{ }^{\dagger \dagger} p<0.01 \mathrm{vs}$ $\mathrm{MN} ;{ }^{\star} p<0.05,{ }^{*} p<0.01$ vs $\mathrm{H} \rightarrow$ $\mathrm{N} ;{ }^{\S} p<0.05,{ }^{\S} p<0.01 \mathrm{vs} \mathrm{H} \rightarrow \mathrm{N}+$ In $\mathrm{NC} ;{ }^{\circledR \oplus} p<0.01$ vs $\mathrm{H} \rightarrow \mathrm{N}+$ $23 \mathrm{~b}$ In
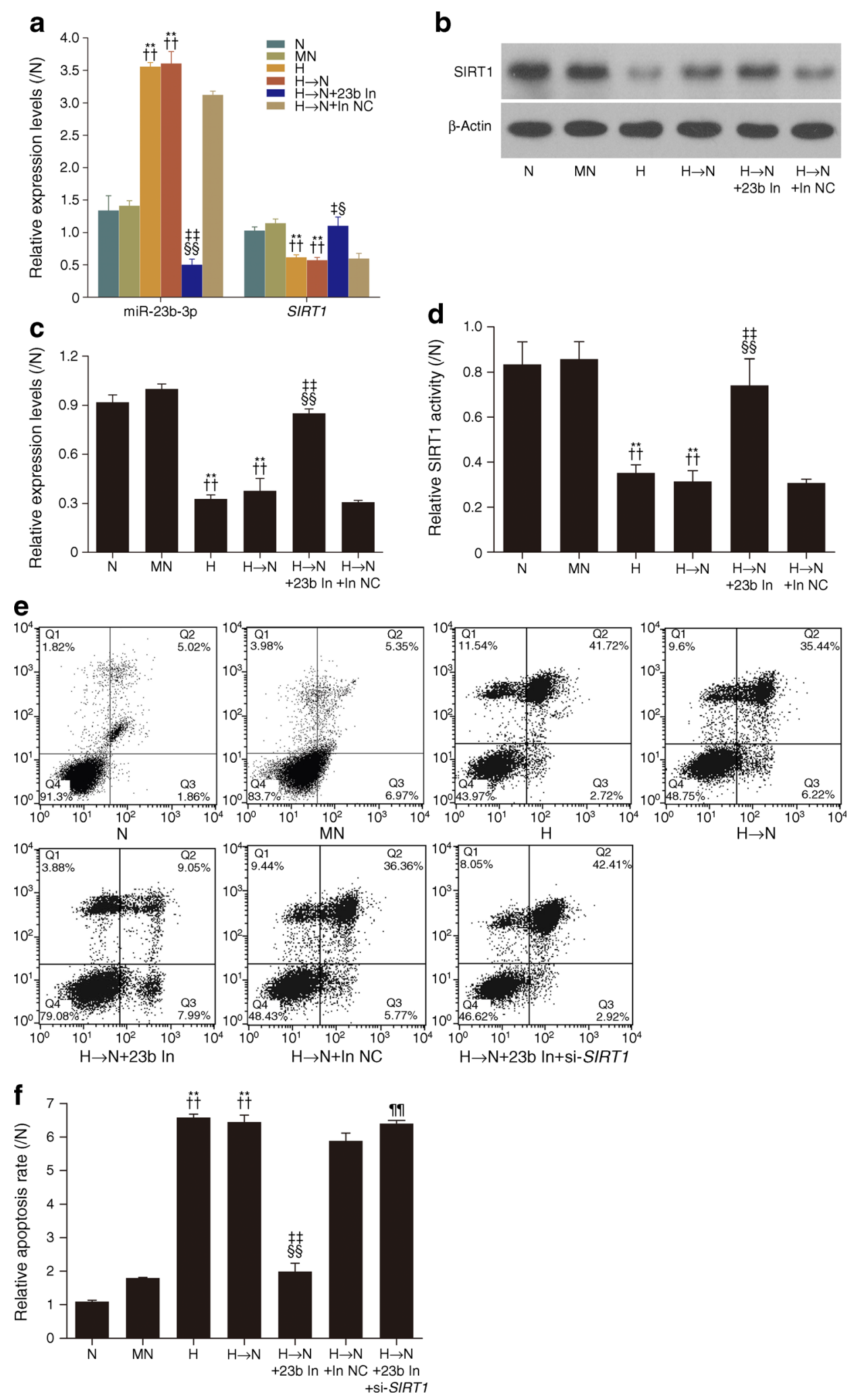

increased 6 months later in STZ-induced diabetic rats [32], suggesting the persistent activation of inflammatory signalling pathways. Using the cell and rat models of metabolic memory induced by high glucose established in our previous study, we demonstrated that SIRT1 activation suppressed the cellular metabolic memory of high glucose in retinal endothelial cells by inhibiting the inflammatory gene $N F-\kappa B$ (also known as Rela) and the apoptosis gene Bax [14]. In the present study, 

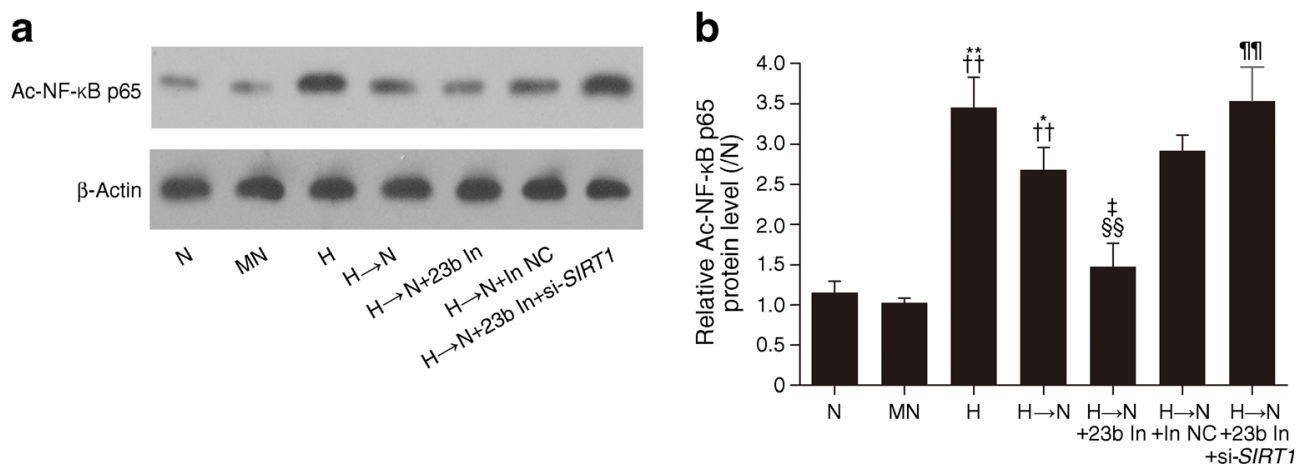

Fig. 4 miR-23b-3p increases Ac-NF-kB expression through inhibiting SIRT1 in HRECs after culture in high- followed by normal-glucose conditions. Western blot (a) and quantification (b) of Ac-NF- $\mathrm{kB}$ expression in the following cell treatment groups: $\mathrm{N}, \mathrm{MN}, \mathrm{H}, \mathrm{H} \rightarrow \mathrm{N}, \mathrm{H} \rightarrow \mathrm{N}+$ miR23b-3p inhibitor $(\mathrm{H} \rightarrow \mathrm{N}+23 \mathrm{~b}$ In), $\mathrm{H} \rightarrow \mathrm{N}+$ miR-23b-3p inhibitor $\mathrm{NC}$

we identified another level of regulation for glucose-induced SIRT1 downregulation and its downstream effects mediated through the alteration of miR-23b-3p.

Produced as small, non-protein-coding RNAs, miRNAs are endogenous regulators that play significant roles in many cellular processes [33,34]. Accumulating evidence has recently illustrated the role of miRNAs in the pathogenesis of diabetes and its complications, including diabetic retinopathy $[15,35,36]$. The miRNA miR-125b plays a role in the dysregulation of Suv39h1 and associated chromatin H3K9me3 related to the increased expression of inflammatory genes in the vascular smooth muscle cells of diabetic mice. These results revealed an miRNA-related mechanism for sustained diabetic complications even after achieving glycaemic control [37].

We identified another miRNA, miR-23b-3p, as an important regulator of HRECs in diabetic retinopathy. Our results describe the role of miR-23b-3p in SIRT1 downregulation in HRECs, which also mediates Ac-NF-KB upregulation in the metabolic memory induced by high glucose in HRECs. We investigated the mechanisms contributing to this regulation at multiple levels. After the initial identification of miR-23b-3p upregulation in HRECs in the miRNA microarray, we used an miR-23b-3p antagomir to identify its biological significance in vitro. We examined the functional significance of this mechanism in vivo using an established animal model of diabetic retinopathy. Following exposure to hyperglycaemia, miR-23b-3p is expressed in microvessel endothelial cells and the cells of the outer nuclear layers (Fig. 5b). The regulation of SIRT1 by miR-23b-3p may not be specific to endothelial cells. Further studies are necessary to address such issues.

Acetylation of NF- $\mathrm{KB}$ increases significantly in diabetesrelated vascular complications and is required for transcriptional activation of its target genes [38]. Under normal physiological conditions, there is a balance between the histone acetylation and deacetylation of NF- $\mathrm{kB}$ mediated by the histone acetyltransferases (HATs) and histone deacetylases

$(\mathrm{H} \rightarrow \mathrm{N}+\mathrm{In} \mathrm{NC})$ and $\mathrm{H} \rightarrow \mathrm{N}+$ miR-23b-3p inhibitor and SIRT1 siRNA $(\mathrm{H} \rightarrow \mathrm{N}+23 \mathrm{~b}$ In + si-SIRT1). Results are mean $\pm \mathrm{SD}$ and fold relative to the level in one N HREC group $(/ \mathrm{N})$ from three separate experiments; ${ }^{*} p<0.05,{ }^{* *} p<0.01$ vs $\mathrm{N} ;{ }^{\dagger \dagger} p<0.01$ vs $\mathrm{MN} ;{ }^{*} p<0.05$ vs $\mathrm{H} \rightarrow \mathrm{N}$; ${ }^{\S \S} p<0.01$ vs $\mathrm{H} \rightarrow \mathrm{N}+\mathrm{In} \mathrm{NC} ;{ }^{{ }^{\top}} p<0.01$ vs $\mathrm{H} \rightarrow \mathrm{N}+23 \mathrm{~b}$ In

(HDACs), respectively [39]. High glucose could increase the recruitment of HATs and decrease the association of HDACs [40], improving histone acetylation and NF- $\mathrm{KB}$ acetylation [41]. Chromatin histone modifications and enhanced NF-kB transcription activity allow increased binding of NF- $\mathrm{kB}$ to regulate inflammatory and diabetes-related genes [42].

The mechanism of stable NF- $\mathrm{kB}$ acetylation in metabolic memory has not been elucidated. As an epigenetic regulatory mechanism based on the above results, [43], we postulated that the chromatin histone modification mediated by transient hyperglycaemia would continue to recruit HATs and may be one of the mechanisms resulting in the continuous acetylation of NF-KB, which needs to be further investigated.

A class III histone deacetylase, SIRT1 inhibits NF- $\mathrm{kB}$ transcription by directly deacetylating the RelA/p 65 protein at Lys310 and mediates anti-inflammatory effects in several pathophysiological processes. Yeung et al demonstrated that SIRT1-mediated deacetylation of Lys310 inhibits the transactivation of the RelA/p65 subunit and consequently suppresses NF-kB-dependent gene expression [39]. Liu et al reported that chronic inflammation stimulates the accumulation of SIRT1 in the promoter regions of cytokine genes and then induces deacetylation at the RelA/p65 subunit of the NF-kB complex [44]. Moreover, SIRT1-mediated deacetylation of NF-KB is crucially involved in the resolution of inflammation and metabolic disorders [45-47]. Our present study confirms the above findings and shows that SIRT1 represses highglucose-induced cellular metabolic memory, at least in part through the deacetylation of NF-kB. Moreover, this effect can be regulated by an upstream miRNA, miR-23b-3p, which targets SIRT1 in diabetic retinopathy.

miRNAs frequently form positive feedback loops as they are themselves regulated by transcription factors that they directly or indirectly target $[48,49]$. Such self-reinforcing circuits result from initiating events and are maintained by selfsustained feedback loops in the absence of the initiating signal [24]. 


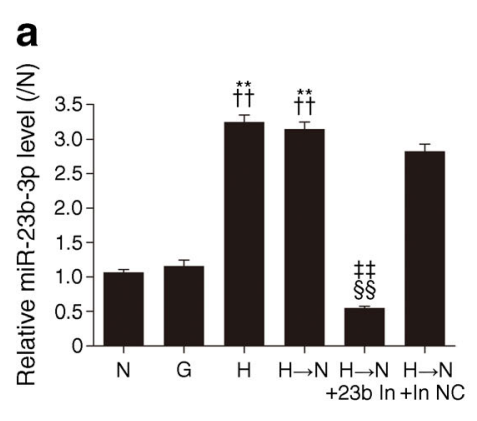

C

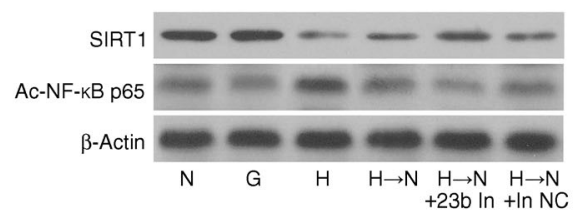

e
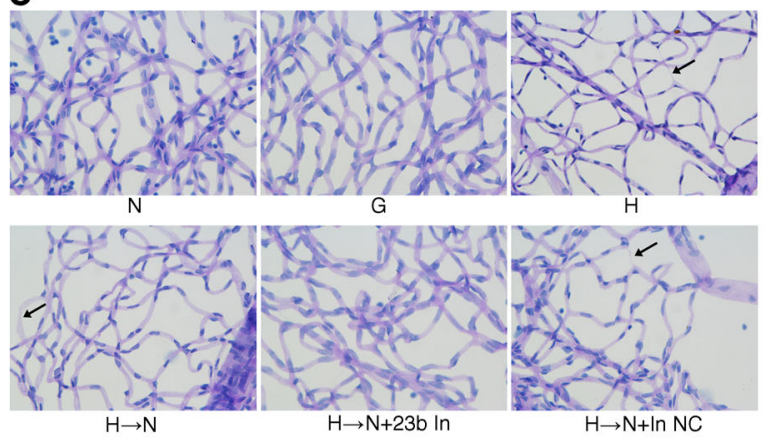

g

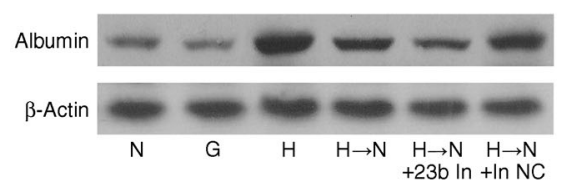

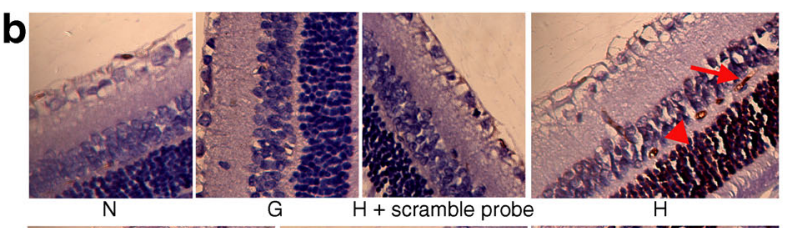
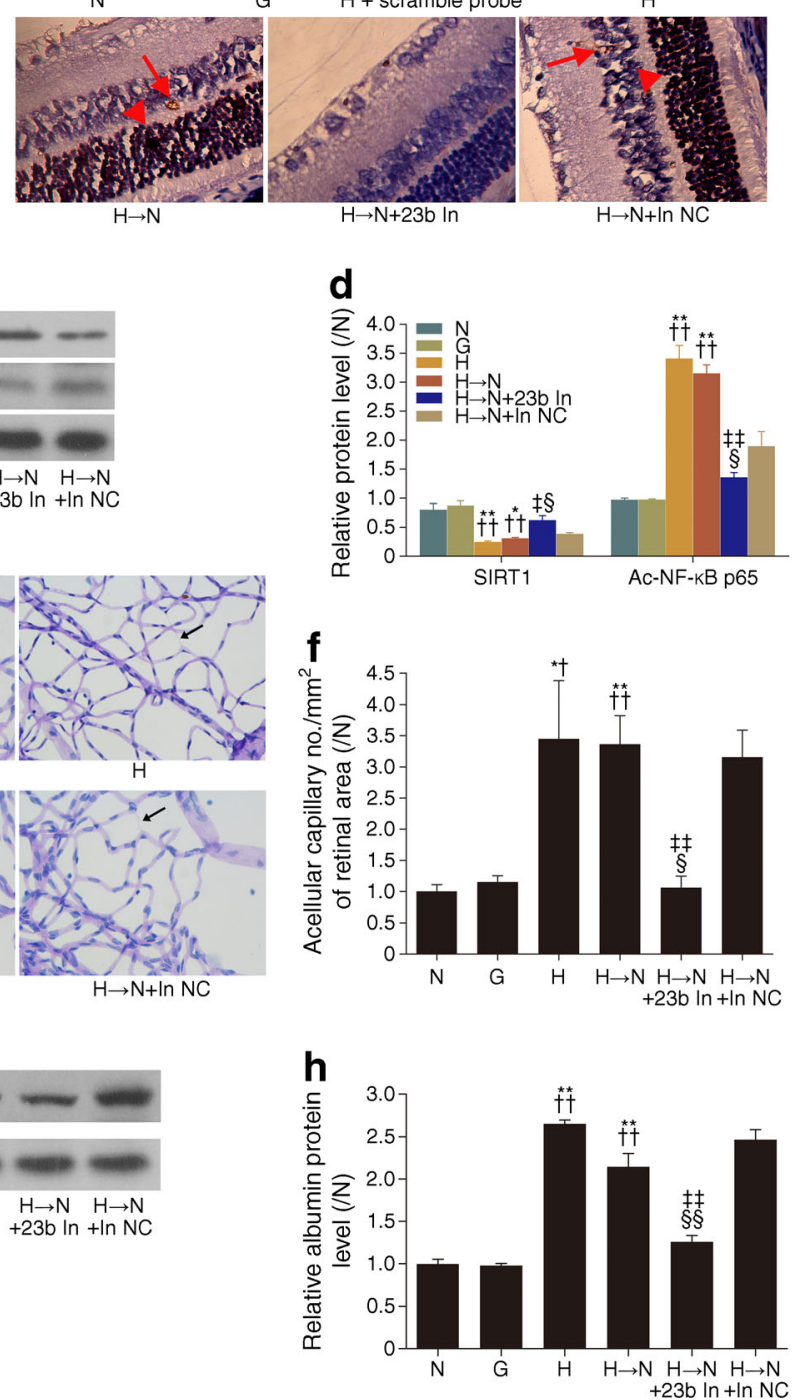

photomicrographs (e) and quantification (f) of acellular capillaries in trypsin-digested retinal blood vessels obtained from the six groups mentioned above (magnification: $\times 400$ ). All preparations were stained with periodic acid-Schiff's reagent (PAS) and haematoxylin. Arrows indicate an acellular capillary. (g) Western blot and (h) quantification of leaked albumin from the retina lysates of the six groups. A representative experiment of the three is shown. Results are mean $\pm \mathrm{SD}$ and fold relative to the level in one $\mathrm{N}$ rat group $(/ \mathrm{N})$ from three separate experiments; ${ }^{*} p<0.05, * * p<0.01$ vs N; ${ }^{\dagger} p<0.05,{ }^{\dagger \dagger} p<0.01$ vs G; ${ }^{\star} p<0.05$, ${ }^{\mathrm{H}} p<0.01$ vs $\mathrm{H} \rightarrow \mathrm{N} ;{ }^{\S} p<0.05,{ }^{\S}{ }_{p} p<0.01$ vs $\mathrm{H} \rightarrow \mathrm{N}+\mathrm{In} \mathrm{NC}$

miR-23b-3p-mediated repression of SIRT1 and controls NF-KB and chronic inflammation (Fig. 7). This potential miR-23b-3p/SIRT1/NF-kB feedback loop may play a key role in establishing and maintaining metabolic memory in diabetic retinopathy. As RNA-based therapies hold potential advantages because of their specificity, targeting specific miRNAs 
a

Chr:9

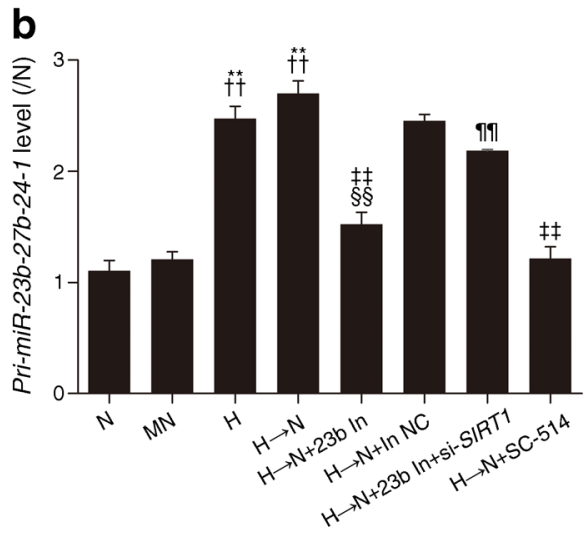

Fig. 6 Promoter binding of p65 transactivates the miR-23b gene in HRECs after culture in $\mathrm{H} \rightarrow \mathrm{N}$ conditions. (a) A schematic diagram showing the structure of the $m i R-23 b-27 b-24-1$ gene, with the positions of the potential NF- $\mathrm{kB}$ binding sites and the primer set for ChIP assay in its promoter. Hsa-, human. (b) Quantitative RT-PCR analysis showed the increased expression of pri-miR23b-27b-24-1 in HRECs of $\mathrm{H}$ and $\mathrm{H} \rightarrow$ $\mathrm{N}$ groups, which was inhibited by miR-23b inhibitor through the SIRT1dependent pathway $(\mathrm{H} \rightarrow \mathrm{N}+$ miR-23b-3p inhibitor $(\mathrm{H} \rightarrow \mathrm{N}+23 \mathrm{~b}$ In $)$ and $\mathrm{H} \rightarrow \mathrm{N}+$ miR-23b-3p inhibitor and SIRT1 siRNA $(\mathrm{H} \rightarrow \mathrm{N}+23 \mathrm{~b} \mathrm{In}+\mathrm{si}-$

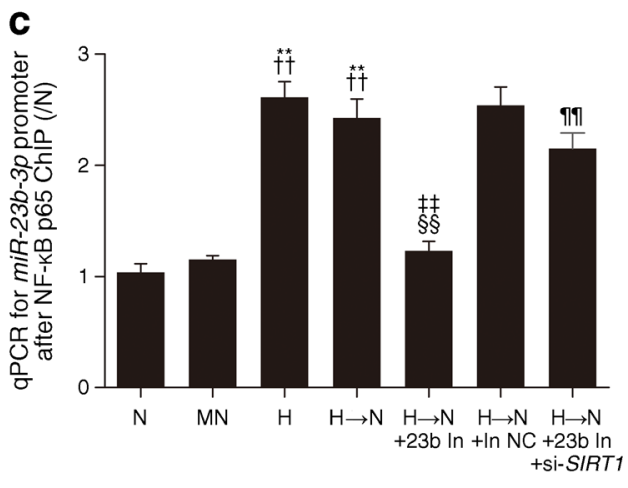

SIRT1) and the NF-kB inhibitor SC-512. (c) ChIP analysis revealed increased binding of $\mathrm{p} 65$ to the potential binding site of the $m i R-23 b-27 b$ 24-1 gene promoter element in HRECs of group $\mathrm{H}$ or $\mathrm{H} \rightarrow \mathrm{N}$, and the increased binding was suppressed by miR-23b inhibitor through the SIRT1-dependent pathway $(\mathrm{H} \rightarrow \mathrm{N}+23 \mathrm{~b}$ In and $\mathrm{H} \rightarrow \mathrm{N}+23 \mathrm{~b}$ In + si-SIRT1). Results are mean \pm SD and fold relative to the level in one N HREC group (/N) from three separate experiments; ${ }^{* *} p<0.01$ vs $\mathrm{N},{ }^{\dagger \dagger} p<0.01$ vs MN; ${ }_{\mathrm{At}} p<0.01$ vs $\mathrm{H} \rightarrow \mathrm{N} ;{ }^{\S} p<0.01$ vs $\mathrm{H} \rightarrow \mathrm{N}+\mathrm{In} \mathrm{NC} ;{ }^{q \pi} p<0.01$ vs $\mathrm{H} \rightarrow \mathrm{N}+$ $23 \mathrm{~b}$ In. FP, forward primer; RP, reverse primer
Fig. 7 Schematic representation of the proposed NF- $\mathrm{kB} / \mathrm{miR}-23 \mathrm{~b}-$ $3 p /$ SIRT1 feedback loop and its potential involvement in the cellular metabolic memory of high glucose in diabetic retinopathy. On one hand, high glucose stimulates miR-23b-3p expression by increasing the positive transcription factor Ac$\mathrm{NF}-\mathrm{kB}$. On the other hand, miR$23 b-3 p$ increases the abundance of Ac-NF- $\mathrm{kB}$ by reducing the negative regulator SIRT1. HG, high glucose; RISC, RNAinduced silencing complex. miR$23 b-3 p$ is a core component of RISC and is essential for recognising SIRT1 mRNA, resulting in its cleavage and inhibition of translation by other components included in RISC

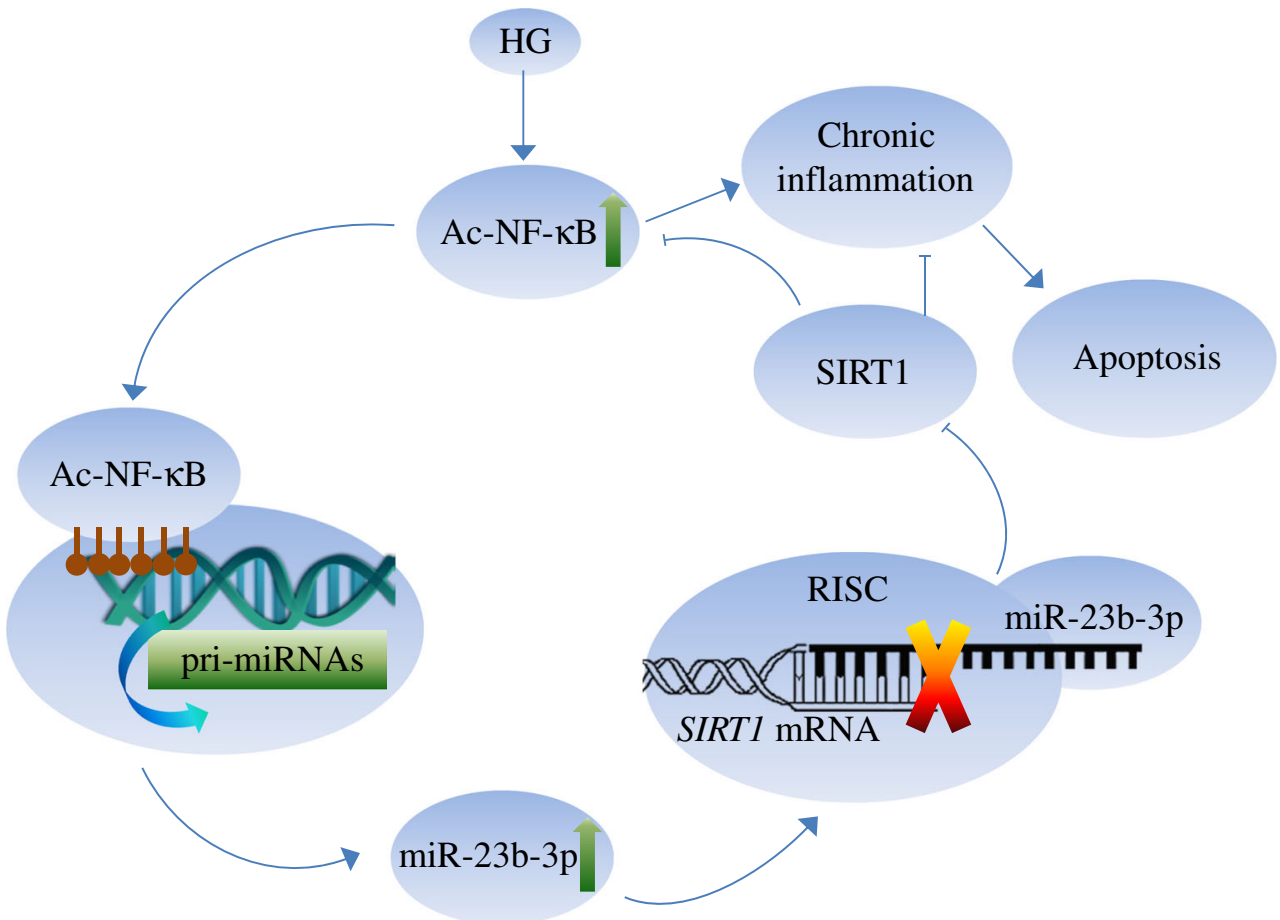


in combination therapy may offer a promising strategy for halting the development of diabetic retinopathy and other diabetes complications.

Funding This work was supported by grants from the National Natural Science Foundation of China (numbers 81470643, 81271032, 81070739 and 30872828) and the National Key Basic Research Program (2010CB535006).

Duality of interest The authors declare that there is no duality of interest associated with this manuscript.

Contribution statement SZ, HCh and ZZ conceived and designed the experiments. SZ, HCh, ZZ, TL, JL, QL, CH, NW, QQ, HCa and XX contributed to the acquisition of data. SZ, HCh, ZZ and XX analysed and interpreted the data. SZ, HCh and ZZ contributed to drafting the article. All authors have revised the manuscript critically for important intellectual content and approved the final version to be published. $\mathrm{HCh}$ and $\mathrm{ZZ}$ are responsible for the integrity of the work as a whole.

\section{References}

1. Brownlee M (2001) Biochemistry and molecular cell biology of diabetic complications. Nature 414:813-820

2. Reddy MA, Zhang E, Natarajan R (2015) Epigenetic mechanisms in diabetic complications and metabolic memory. Diabetologia 58:443-455

3. The Diabetes Control and Complications Trial Research Group/ Epidemiology of Diabetes Interventions and Complications Research Group (2002) Effect of intensive therapy on the microvascular complications of type 1 diabetes mellitus. JAMA 287:2563-2569

4. Ola MS, Nawaz MI, Siddiquei MM, Al-Amro S, Abu El-Asrar AM (2012) Recent advances in understanding the biochemical and molecular mechanism of diabetic retinopathy. J Diabetes Complicat 26:56-64

5. Kowluru RA, Chan PS (2010) Metabolic memory in diabetes from in vitro oddity to in vivo problem: role of apoptosis. Brain Res Bull 81:297-302

6. Zhong Q, Kowluru RA (2013) Epigenetic modification of Sod2 in the development of diabetic retinopathy and in the metabolic memory: role of histone methylation. Invest Ophthalmol Vis Sci 54:244-250

7. Baker RG, Hayden MS, Ghosh S (2011) NF-kappaB, inflammation, and metabolic disease. Cell Metab 13:11-22

8. Caron AZ, He X, Mottawea W et al (2014) The SIRT1 deacetylase protects mice against the symptoms of metabolic syndrome. FASEB J 28:1306-1316

9. Khan SA, Sathyanarayan A, Mashek MT, Ong KT, WollastonHayden EE, Mashek DG (2015) ATGL-catalyzed lipolysis regulates SIRT1 to control PGC-1alpha/PPAR-alpha signaling. Diabetes 64:418-426

10. Gilbert RE, Thai K, Advani SL et al (2015) SIRT1 activation ameliorates hyperglycaemia by inducing a torpor-like state in an obese mouse model of type 2 diabetes. Diabetologia 58:819-827

11. Cai W, Ramdas M, Zhu L, Chen X, Striker GE, Vlassara H (2012) Oral advanced glycation endproducts (AGEs) promote insulin resistance and diabetes by depleting the antioxidant defenses AGE receptor-1 and sirtuin 1. Proc Natl Acad Sci U S A 109:1588815893
12. Yang L, Zhang J, Yan C et al (2012) SIRT1 regulates CD40 expression induced by TNF-alpha via NF-kB pathway in endothelial cells. Cell Physiol Biochem 30:1287-1298

13. Wang W, Bai L, Qiao $\mathrm{H}$ et al (2014) The protective effect of fenofibrate against TNF-alpha-induced CD40 expression through SIRT1-mediated deacetylation of NF-kappaB in endothelial cells. Inflammation 37:177-185

14. Zheng Z, Chen H, Li J et al (2012) Sirtuin 1-mediated cellular metabolic memory of high glucose via the LKB1/AMPK/ROS pathway and therapeutic effects of metformin. Diabetes 61:217228

15. Wu JH, Gao Y, Ren AJ et al (2012) Altered microRNA expression profiles in retinas with diabetic retinopathy. Ophthalmic Res 47: 195-201

16. Guay C, Regazzi R (2013) Circulating microRNAs as novel biomarkers for diabetes mellitus. Nat Rev Endocrinol 9:513-521

17. Arner P, Kulyte A (2015) MicroRNA regulatory networks in human adipose tissue and obesity. Nat Rev Endocrinol 11:276-288

18. Ito T, Yagi S, Yamakuchi M (2010) MicroRNA-34a regulation of endothelial senescence. Biochem Biophys Res Commun 398:735-740

19. Ramachandran D, Roy U, Garg S, Ghosh S, Pathak S, KolthurSeetharam U (2011) Sirt1 and mir-9 expression is regulated during glucose-stimulated insulin secretion in pancreatic beta-islets. FEBS J 278:1167-1174

20. Mortuza R, Feng B, Chakrabarti S (2014) miR-195 regulates SIRT1-mediated changes in diabetic retinopathy. Diabetologia 57:1037-1046

21. Li JH, Liu S, Zhou H, Qu LH, Yang JH (2014) starBase v2.0: decoding miRNA-ceRNA, miRNA-ncRNA and protein-RNA interaction networks from large-scale CLIP-Seq data. Nucleic Acids Res 42:D92-D97

22. Ha M, Kim VN (2014) Regulation of microRNA biogenesis. Nat Rev Mol Cell Biol 15:509-524

23. Zhou R, Hu G, Gong AY, Chen XM (2010) Binding of NF-kappaB p65 subunit to the promoter elements is involved in LPS-induced transactivation of miRNA genes in human biliary epithelial cells. Nucleic Acids Res 38:3222-3232

24. Rokavec M, Oner MG, Li H et al (2014) IL-6R/STAT3/miR-34a feedback loop promotes EMT-mediated colorectal cancer invasion and metastasis. J Clin Invest 124:1853-1867

25. Xiang Y, Cheng J, Wang D et al (2015) Hyperglycemia repression of miR-24 coordinately upregulates endothelial cell expression and secretion of von Willebrand factor. Blood 125:3377-3387

26. The Diabetes Control and Complications Trial Research Group (1995) The effect of intensive diabetes therapy on the development and progression of neuropathy. Ann Intern Med 122:561-568

27. The Diabetes Control and Complications Trial Research Group/ Epidemiology of Diabetes Interventions and Complications Research Group (2003) Sustained effect of intensive treatment of type 1 diabetes mellitus on development and progression of diabetic nephropathy: the Epidemiology of Diabetes Interventions and Complications (EDIC) study. JAMA 290:2159-2167

28. Noonan JE, Jenkins AJ, Ma JX, Keech AC, Wang JJ, Lamoureux EL (2013) An update on the molecular actions of fenofibrate and its clinical effects on diabetic retinopathy and other microvascular end points in patients with diabetes. Diabetes 62:3968-3975

29. Aiello LP (2014) Diabetic retinopathy and other ocular findings in the diabetes control and complications trial/epidemiology of diabetes interventions and complications study. Diabetes Care 37:17-23

30. Reidy K, Kang HM, Hostetter T, Susztak K (2014) Molecular mechanisms of diabetic kidney disease. J Clin Invest 124:2333-2340

31. Cheung CY, Ikram MK, Klein R, Wong TY (2015) The clinical implications of recent studies on the structure and function of the retinal microvasculature in diabetes. Diabetologia 58:871-885 
32. Kowluru RA, Chakrabarti S, Chen S (2004) Re-institution of good metabolic control in diabetic rats and activation of caspase- 3 and nuclear transcriptional factor (NF-kappaB) in the retina. Acta Diabetol 41:194-199

33. Bartel DP (2009) MicroRNAs: target recognition and regulatory functions. Cell 136:215-233

34. Kantharidis P, Wang B, Carew RM, Lan HY (2011) Diabetes complications: the microRNA perspective. Diabetes 60:1832-1837

35. Kovacs B, Lumayag S, Cowan C, Xu S (2011) MicroRNAs in early diabetic retinopathy in streptozotocin-induced diabetic rats. Invest Ophthalmol Vis Sci 52:4402-4409

36. Lorenzen J, Kumarswamy R, Dangwal S, Thum T (2012) MicroRNAs in diabetes and diabetes-associated complications. RNA Biol 9:820-827

37. Villeneuve LM, Kato M, Reddy MA, Wang M, Lanting L, Natarajan R (2010) Enhanced levels of microRNA-125b in vascular smooth muscle cells of diabetic $\mathrm{db} / \mathrm{db}$ mice lead to increased inflammatory gene expression by targeting the histone methyltransferase Suv39h1. Diabetes 59:2904-2915

38. Liu R, Zhong Y, Li X et al (2014) Role of transcription factor acetylation in diabetic kidney disease. Diabetes 63:2440-2453

39. Yeung F, Hoberg JE, Ramsey CS et al (2004) Modulation of NFkappaB-dependent transcription and cell survival by the SIRT1 deacetylase. EMBO J 23:2369-2380

40. Miao F, Gonzalo IG, Lanting L, Natarajan R (2004) In vivo chromatin remodeling events leading to inflammatory gene transcription under diabetic conditions. J Biol Chem 279:18091-18097
41. Ruderman NB, Williamson JR, Brownlee M (1992) Glucose and diabetic vascular disease. FASEB J 6:2905-2914

42. Lee W, Lee SY, Son YJ, Yun JM (2015) Gallic acid decreases inflammatory cytokine secretion through histone acetyltransferase/ histone deacetylase regulation in high glucose-induced human monocytes. J Med Food 18:793-801

43. Cooper ME, El-Osta A (2010) Epigenetics: mechanisms and implications for diabetic complications. Circ Res 107:1403-1413

44. Liu TF, Yoza BK, El Gazzar M, Vachharajani VT, McCall CE (2011) NAD+-dependent SIRT1 deacetylase participates in epigenetic reprogramming during endotoxin tolerance. J Biol Chem 286:9856-9864

45. Yu J, Auwerx J (2010) Protein deacetylation by SIRT1: an emerging key post-translational modification in metabolic regulation. Pharmacol Res 62:35-41

46. Yao H, Rahman I (2012) Perspectives on translational and therapeutic aspects of SIRT1 in inflammaging and senescence. Biochem Pharmacol 84:1332-1339

47. Xie J, Zhang X, Zhang L (2013) Negative regulation of inflammation by SIRT1. Pharmacol Res 67:60-67

48. Iorio MV, Croce CM (2012) microRNA involvement in human cancer. Carcinogenesis 33:1126-1133

49. Ebert MS, Sharp PA (2012) Roles for microRNAs in conferring robustness to biological processes. Cell 149:515-524 